\title{
KOMMUNIKATION DURCH HERRSCHAFTSZEICHEN: RÖMISCHES MILITÄR UND RÖMISCHE AMTSTRÄGER IN DEN PROVINZEN
}

\author{
Werner Eck
}

Herrschaft braucht Kommunikation mit denen, die beherrscht werden, und sie braucht Zeichen, mit denen dargestellt werden kann, wer die Herrschaft ausübt und wie diejenigen sie sehen, über die sie ausgeübt wird. Das gilt auf allen Ebenen, auf der staatlichen wie etwa auch auf der von Organisationen. In der langen Zeitspanne, in der Eugen GerstenMAIER als Präsident des Deutschen Bundestages amtierte, suchte er innerhalb des Parlaments einen Stil zu entwickeln, in dem sich die parlamentarische Demokratie der Bundesrepublik ausdrücken sollte. Steingewordenes Zeugnis seines Wirkens ist in Bonn bis heute das ehemalige Abgeordnetenhochhaus, der ,Lange Eugen', in dem die Mitglieder des demokratisch gewählten Parlaments ihre Büros hatten. Das Gebäude erhob sich weit über alle Ministerien. Dies sollte Zeichen sein. Gleichzeitig aber initiierte er auch die Regel, dass zu Beginn einer Sitzung des Bundestags, wenn der Präsident den Saal betrat, ein Amtsdiener dem Präsidenten vorausging und dabei verkündete: „Der Präsident!“. Zeichenhaft und für alle wahrnehmbar sollte dadurch deutlich werden, dass der höchste Repräsentant des Parlaments anwesend sei und die Arbeit des Parlaments beginnen könne.

Auf ganz anderer Ebene konnte ich im Juni 2007 ein ähnliches Zeremoniell beobachten. An der Universität Nijmegen hielt Lukas DE BLOIS, der Initiator unseres International Network 'The Impact of Empire', seine Abschiedsvorlesung. Als das große Publikum in der Aula Platz genommen hatte, begann der Einzug der Professoren im Talar, angeführt von einem Universitätsbediensteten, der einen Stab mit silbernen Knauf trug, als Insignie des Akademischen Regimes. Ihm folgten der Rektor mit der Amtskette, der Dekan der Fakultät, der Emeritus selbst und darauf die anderen Professoren. Durch Zeichen und durch ritualisiertes Handeln war die akademische Ordnung 
zwischen Lehrenden und Lernenden, aber auch innerhalb der Lehrenden deutlich geworden.

Was hier im Rahmen relativ kleiner, fast noch überschaubarer Personengruppen gilt, ist von weit höherer Bedeutung für große Einheiten, etwa für eine Armee oder für Großreiche wie das Imperium Romanum, das im Zentrum unseres Kolloquiums steht. Gerade solche Großorganisationen brauchen Zeichen oder auch Rituale, in denen sich die gewollte oder als fraglos vorausgesetzte Ordnung manifestiert. So ist zu fragen, wie innerhalb dieses römischen Herrschaftsraumes sichtbar gemacht wurde, zu wem man gehörte oder gehören musste, auch wenn man es nicht wollte, und wem man unterstand. Denn Rom hatte sein Imperium größtenteils mit Gewalt erobert, wodurch ein gewaltiges und komplexes Gebilde entstanden war. Weit größer als die heutige Europäische Union umfasste es einen Raum von rund 5 Millionen $\mathrm{km}^{2}$ Herrschaftsfläche. Vom äußersten Norden Englands bis zur Südgrenze der Provinz Ägypten bei Syene erstreckte sich der Herrschaftsraum über eine Entfernung von rund $4000 \mathrm{~km}$; von der Atlantikküste Portugals bis Armenien und der Provinz Mesopotamia im Osten betrug die Entfernung sogar mehr als $5000 \mathrm{~km}$. Die Völker, Stämme und Poleis, die in dieser Einheit zusammengefasst waren, unterschieden sich in hohem Maße nach Religion, Sprache sowie politischer, sozialer und wirtschaftlicher Struktur. Rom beließ ihnen allen ein hohes $\mathrm{Ma} ß$ an innerer Autonomie, in die nur in Maßen eingegriffen wurde. Dennoch musste immer wieder erkannt und immer wieder wahrgenommen werden, wem die lokalen Einheiten oder auch die Individuen unterstanden oder wem sie zugehörten.

Seit Augustus gehorchte das Reich einem einheitlichen Willen. Doch der Herrscher war fern. Die meisten Bewohner des Imperiums haben den Kaiser nie persönlich zu Gesicht bekommen. Das Bild, das sie sich möglicherweise von ihm machten, stammte von den Münzen, die jeder in die Hand bekommen konnte, und den Statuen, mit denen der jeweilige Herrscher in den einzelnen Gemeinden bildlich präsent war. Deutlich wird dies in der Frage, die Jesus nach Markus 12, 16 mit Verweis auf einen Denar an die Pharisäer richtete ${ }^{1}$ : „Wessen ist dieses Bild und die Aufschrift?“, und in deren Ant-

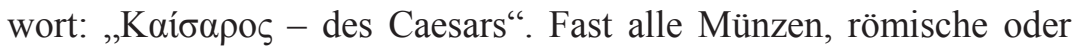

\footnotetext{
${ }^{1}$ Vgl. Matthäus 22.15-22.
} 
lokale, trugen das Porträt des Kaisers. ${ }^{2}$ Selbst strenggläubige Juden konnten sich dem kaum entziehen.

Der Kaiser blieb aber im Allgemeinen der ferne Herrscher, den nur wenige von Angesicht zu Angesicht erlebten; noch weniger Menschen aus den Provinzen traten mit ihm in persönlichen Kontakt. Der Masse der Provinzbewohner erfuhr Rom und den Kaiser durch andere Zeichen - durch Zeichen, die auf die herrschende Macht verwiesen, und vor allem durch Personen, die diese Macht repräsentierten. Es waren die römischen Magistrate, die die Provinzen regierten. Traian formulierte in einem Brief an seinen Legaten Plinius sehr bewusst, der Senator solle an seiner Statt den Provinzialen gegenübertreten: electum te esse, qui ad eosdem mei loco mittereris. ${ }^{3}$ Die Provinzmagistrate erschienen unter wechselnden Formen und mit unterschiedlichen Bezeichnungen: proconsules, legati Augusti pro praetore, praefecti, procuratores. Doch für die Untertanen machte es keinen grundsätzlichen Unterschied, ob die Repräsentanten Roms als senatorische Prokonsuln, als Legaten im Dienste des Herrschers oder als ritterliche Präsidialprokuratoren agierten. Ihre gemeinsame Aufgabe war es, Ruhe in den Provinzen zu gewährleisten, den Rechtsfrieden zu wahren und den Einzug der Steuern direkt oder indirekt zu sichern. Entsprechend waren ihre Rechte formuliert und auch die äußeren Formen gestaltet, in denen die römische Macht sichtbar in Erscheinung trat. Dies ließe sich an vielen Provinzen exemplifizieren, etwa an Germania inferior, wo in der colonia Claudia Ara Agrippinensium eines der wenigen Beispiele für eine Statthalterresidenz der hohen Kaiserzeit zu einem relativ größeren Teil erhalten ist. ${ }^{4}$ Man könnte auch an Dakien denken, wo in der colonia Ulpia Traiana Sarmizegetusa unter anderem der Amtssitz des Finanzprokurators der Provinz ergraben wurde, was Einblicke in die Repräsentationsund Kommunikationsformen dieses Funktionsträgers erlaubt. ${ }^{5}$ Auch

\footnotetext{
${ }^{2}$ Eine Ausnahme waren nur die sogenannten Prokuratorenmünzen, die innerhalb Judaeas geprägt wurden; wer sie prägen ließ, ist nicht geklärt.

${ }^{3}$ Plinius Minor, Epistulae 10.18.2.

${ }^{4}$ G. Precht, Baugeschichtliche Untersuchung zum römischen Praetorium in Köln (Köln 1973); W. Eck, Köln in römischer Zeit. Geschichte einer Stadt im Rahmen des Imperium Romanum (Köln 2004), passim.

${ }^{5}$ I. Piso, 'Inschriften von Prokuratoren aus Sarmizegetusa I', Zeitschrift für Papyrologie und Epigraphik 50 (1983), 233 ff.; ders., 'Inschriften von Prokuratoren aus Sarmizegetusa II', Zeitschrift für Papyrologie und Epigraphik 120 (1998), 253 ff.
} 
in Ephesos, der Hauptstadt von Asia, der reichsten Provinz des römischen Ostens, könnte man aufgrund des Inschriftenreichtums manche der Kommunikationsformen zwischen Herrschenden und Beherrschten anhand von konkreten Beispielen verfolgen. ${ }^{6}$ Doch scheint mir zur Zeit keine Provinz mehr als Judaea/Syria Palaestina geeignet zu sein, um ein relativ konkretes und genügend repräsentatives Bild davon zu entwerfen, in welcher Weise und in welchen konkreten Formen römische Amtsträger in den ersten drei Jahrhunderten der Kaiserzeit die Macht des Reiches gegenüber den Provinzbewohnern darstellten und so die Botschaft vermittelten, dass alle Reichsbewohner einer einzigen Herrschaft unterstanden und deren Willen zu erfüllen hatten. Bedingt ist dies zum einen durch die neueren Ausgrabungsergebnisse, speziell in Caesarea, andererseits durch die für eine römische Provinz exzeptionelle literarische Überlieferung, die es erlaubt, Aspekte zu erkennen, die anderswo nicht oder nur in minimalen Reflexen in den Quellen erscheinen.

Schon vor dem Jahr 6 n. Chr. war Judaea Teil des Imperiums, zunächst noch unter der Herrschaft von Klientelfürsten wie Herodes und später seinem Sohn Archelaos und dessen Brüdern. Als Archelaos als Ethnarch nicht mehr länger tragbar erschien, übernahm Augustus die Region in seine direkte Verantwortung. Die Form, die er wählte, war aber nicht die einer eigenständigen Provinz; vielmehr schloss er das - im Übrigen nicht sehr große Gebiet - der Provinz Syria an, dessen Statthalter als legatus Augusti pro praetore den Kaiser vertrat. Es gab also zunächst keine eigenständige Provinz Judaea, sondern nur eine Region Judaea innerhalb der Provinz Syria, die allerdings einem von Augustus selbst ernannten praefectus unterstellt wurde, der dieses Gebiet verwalten sollte, dabei jedoch dem Statthalter Syriens untergeordnet war. ${ }^{7}$ Die Bewohner dieser Region waren auf diese Weise mit zwei römischen Repräsentanten konfrontiert, von denen der eine im fernen Antiochia residierte und nur gelegentlich auch den südlichen Bereich seiner Provinz besuchte, während

${ }^{6}$ Siehe dazu in Kürze: W. Eck, 'Presence, role and significance of Latin in the epigraphy and culture of the Roman Near East', in: H.M. Cotton - R.G. Hoyland J.J. Price - D.J. Wasserstein (Hrsg.), From Hellenism to Islam: Cultural and Linguistic Change in the Roman Near East (Cambridge 2009; im Druck).

${ }^{7}$ Siehe zu dieser Sichtweise nunmehr M. Bernett, Der Kaiserkult in Judäa unter den Herodiern und Römern (Tübingen 2007), $310 \mathrm{ff}$. und ausführlich W. Eck, Rom und Judaea. Fünf Vorträge zur römischen Herrschaft in Palaestina (Tübingen 2007), $23 \mathrm{ff}$. 
der praefectus Iudaeae unmittelbar vor Ort tätig war und seinen Sitz in der Hafenstadt Caesarea nahm. Diese Doppelverwaltung dauerte mit einer kurzen Unterbrechung zwischen 41 und $44 \mathrm{n}$. Chr. bis zum Jahr 66 an, als der große jüdische Aufstand zu einer völligen Neuorganisation führte. Zwar wird auch heute noch öfter behauptet, Judaea sei von Augustus zu einer eigenständigen Provinz gemacht oder spätestens im Jahr 44 als unabhängige Provinz unter einem ritterlichen Prokurator organisiert worden. Wenn man jedoch Josephus in seiner konkreten Berichterstattung und nicht in seiner Terminologie für die römischen Vertreter vor Ort ernst nimmt, dann kann es bei unseren heutigen Kenntnissen kaum einen Zweifel geben, dass Judaea bis 66 n. Chr. ein Teil der Großprovinz Syrien geblieben ist.

Der jüdische Aufstand brachte den Umschwung: Mit Vespasian, der als Konsular nach Judaea gesandt wurde und drei Legionen und entsprechende Hilfstruppen kommandierte, wurden Judaea und die angrenzenden Gebiete aus der Verfügungsgewalt des syrischen Statthalters gelöst. ${ }^{8}$ Nach der Eroberung Jerusalems erscheint dann regelmäßig ein Senator prätorischen Ranges als Statthalter der Provinz, dem auch eine Legion unterstellt war, die legio X Fretensis, welche im zerstörten Jerusalem stationiert wurde, dem ehemaligen religiösen Mittelpunkt und damit dem Machtzentrum des Judentums. An dessen Stelle trat das römische Legionslager - krasser hätte man nicht demonstrieren können, wer hier nun der Herr war und wem auch die jüdischen Bewohner der neuen Provinz zu gehorchen hatten. ${ }^{9}$ Die römische Militärmacht trat an die Stelle des religiösen Zentrums der Juden.

Caesarea aber blieb das politisch-administrative Zentrum wie schon zuvor unter den Präfekten. Die für die römische Herrschaft zentrale Stellung der Stadt wurde allerdings dadurch verstärkt, dass dem von nun an für die Finanzadministration der Provinz zuständigen Prokurator ebenfalls Caesarea als Sitz zugewiesen wurde. Zuvor hatte der Prokurator Syriens von Antiochia aus die Steuern in Judaea eingezogen und sich um die anfallenden Finanzangelegenheiten ge-

\footnotetext{
${ }^{8} \mathrm{Vgl}$. Eck, Rom und Judaea (Anm. 7), $50 \mathrm{f}$.

${ }^{9}$ Bisher ist es nicht gelungen, den Ort, an dem das Lager in Jerusalem errichtet worden war, eindeutig zu bestimmen. Doch ist zu hoffen, dass die großangelegten Grabungen, die zur Zeit stattfinden, darüber endlich Klarheit schaffen.
} 
kümmert. ${ }^{10}$ Zusätzlich wurde aber Caesarea noch dadurch herausgehoben, dass Vespasian die Stadt zu einer römischen Kolonie erhob und damit das römisch-lateinische Element auch im munizipalen Bereich dominieren ließ.

Unter Traian wurde der Status der Provinz insoweit erhöht, als mit der Stationierung einer zweiten Legion bei Caparcotna im Norden der Provinz der Statthalter nun aus den Reihen der Senatoren konsularen Ranges genommen wurde. Er war der oberste Kommandeur aller in der Provinz stationierten Truppen, doch standen von da an zwei senatorische Legionslegaten unter ihm. Dieser Zustand blieb dann mindestens bis in das späte dritte Jahrhundert erhalten. ${ }^{11}$

Seit Pompeius im Jahr 63 v. Chr. vor Jerusalem erschienen war, um seinen politischen Willen mit seinem Heer durchzusetzen, hatten die Führungsschicht und die Bevölkerung Judaeas erlebt, wie Rom durch seine Truppen die eigene Herrschaft konkretisierte. Als sich nach dem Tod von König Herodes im Jahre 4 v. Chr. Sabinus, der Prokurator Syriens, in den Besitz der königlichen Kassen setzen wollte, kam es in Jerusalem zu einem Aufstand, in dessen Folge Quinctilius Varus, der Statthalter der Provinz, mit seinen Legionen in Jerusalem und im ganzen Land eingreifen musste. ${ }^{12} 2000$ Aufständische ließ er ans Kreuz nageln, ${ }^{13}$ eine brutale Demonstration der Herrschaft und eine deutliche Botschaft, wie Rom mit denen verfahren konnte, die sich gegen seine Herrschaft aufzulehnen versuchten. ${ }^{14}$ Da Archelaos von Augustus schließlich doch als Ethnarch eingesetzt wurde, zog sich die römische Legion, die Varus in Jerusalem zurückgelassen hatte, wieder aus der Region zurück. Doch die Drohung, jederzeit wieder von Norden her vorrücken zu können, blieb bestehen. Immerhin lief, wohl auch aus diesen Erfahrungen heraus, die Provinzialisierung im Jahre $6 \mathrm{n}$. Chr. so friedlich ab, dass die Truppen Syriens nicht einzugreifen brauchten. Die kleinen militäri-

\footnotetext{
${ }^{10}$ Dass Judaea auch in fiskalischer Hinsicht zu Syrien gerechnet wurde, wurde beim Tod des Herodes deutlich, als der Prokurator Syriens versuchte, das königliche Erbe direkt zu übernehmen; Josephus, Antiquitates Judaicae 17.221, 252 ff.; PIR ${ }^{2} \mathrm{~S}$ 33. Siehe auch weiter unten im Text.

${ }^{11}$ Eck, Rom und Judaea (Anm. 7), $112 \mathrm{ff}$.

${ }^{12}$ Josephus, Antiquitates Judaicae $17.286 \mathrm{ff}$.

${ }^{13}$ Josephus, Antiquitates Judaicae 17.295.

${ }^{14}$ Auch in der Begnadigung konnte sich allerdings Herrschaft manifestieren; vgl. Matthäus $27.15 \mathrm{ff}$.
} 
schen Einheiten des Archelaos blieben bestehen und wurden dem Befehl des praefectus Iudaeae unterstellt.

Doch in den nachfolgenden Jahrzehnten wurden immer wieder die Legionen Syriens in Marsch gesetzt; nicht nur, weil es in Judaea zu Unruhen kam, sondern auch, um Herrschaftsformen durchzusetzen, die vielen Provinzialen widersinnig erscheinen mussten. So gab Caligula im Jahr 39 den Befehl, seine Statue im Tempel in Jerusalem aufzustellen - ein fremder Gott sollte also im Hause des Gottes der Juden Wohnung nehmen. Der Statthalter Syriens, P. Petronius, wurde damit beauftragt, den Befehl ausführen. Als die jüdische Bevölkerung sich massiv zur Wehr setzte, zog er, obwohl er Caligulas Befehl für falsch hielt, zwei Legionen Syriens in Ptolemais zusammen, um mit ihnen dem Willen des Kaisers Nachdruck zu verleihen. ${ }^{15}$ Dass dies am Ende nicht geschah, war nur dem Umstand zu verdanken, dass Caligula starb, bevor Petronius gezwungen gewesen wäre, die Aufstellung der Statue im Tempel mit militärischer Gewalt durchzusetzen. Ansonsten hätte sich die große jüdische Revolte vermutlich schon 25 Jahre früher ereignet, die in dieser Form erst 66 n. Chr., wiederum nach dem Eingreifen einer römischen Legion in Jerusalem, aufloderte. Als König Agrippa den Juden in Jerusalem vermitteln wollte, was es bedeute, sich gegen die römische Militärmacht aufzulehnen, kam diese Botschaft nicht mehr an.

Die letzte Konsequenz war die Zerstörung Jerusalems und seit 70 n. Chr. die Stationierung einer Legion in dieser Stadt. Nunmehr wurde das gesamte Land mit kleinen Lagern überzogen, in denen Auxiliartruppen und Abteilungen der Jerusalemer Legion die römische Macht präsent hielten. ${ }^{16}$ Die intendierte Wirkung dieser Militärposten kann man sich vielleicht vergegenwärtigen, wenn man die Überreste der Lager betrachtet, die von Flavius Silva bei Masada errichtet wurden, als dort die letzten Überreste der Aufständischen ausgerottet werden sollten. Dennoch war am Ende die Auswirkung der konkreten Präsenz der Militärmacht gering. So verlegte Traian spätestens gegen Ende seiner Regierung eine zweite Legion in die Provinz und verdoppelte die Zahl der Hilfstruppen auf drei Alen und 12 Kohor-

\footnotetext{
${ }^{15}$ Josephus, Antiquitates Judaicae $18.261 \mathrm{ff}$.

${ }^{16}$ B. Isaac, The Limits of Empire: The Roman Army in the East (Oxford 1993), $427 \mathrm{ff}$.
} 
ten, darunter drei cohortes milliariae. ${ }^{17}$ Das waren mit den beiden Legionen zusammen nahezu 20.000 Mann. Keine andere Provinz kannte im Verhältnis zu ihrer Größe - sicher weniger als 16.000 qkm. - eine solche Militärpräsenz. ${ }^{18}$ Im Jahr 132 explodierte die Provinz jedoch erneut, und der folgende fast vierjährige Krieg gegen die Einheiten Bar Kochbas kostete Hunderttausende von Opfern auf römischer, aber noch weit mehr auf jüdischer Seite. ${ }^{19}$ Erst diesmal wurde die Botschaft, dass Rom eine Provinz, die es einmal in Besitz genommen hatte, nicht mehr hergab, in ihrer ganzen und harten Realität auch von der jüdischen Bevölkerung erfasst. Roms Herrschaft durfte niemand in Frage stellen. Das drückte sich auch in den ornamenta triumphalia aus, die Hadrian den drei Statthaltern von Judaea, Syria und Arabia verlieh: Sex. Iulius Severus, der aus der Provinz Dalmatien stammte, Q. Poblicius Marcellus aus dem italischen Aquileia und T. Haterius Nepos aus Fulginiae in Umbrien. Alle drei hatten durch ihre erfolgreiche Kampfführung gegen die Aufständischen das Prestige Roms in der Region gerettet. ${ }^{20}$

Wohl kein Bevölkerungsteil und keine Provinz haben so lange und mit solchem Widerstand auf diese Botschaft von der Ewigkeit der römischen Herrschaft reagiert und sich ihr so wenig angepasst wie das jüdische Volk. Doch im Grunde hat Rom durch seine Amtsträger und vor allem durch seine Truppen diese Botschaft in allen Provinzen verkündet. König Agrippa, auf den schon verwiesen wurde, hatte diese Botschaft verstanden, war damit aber in Judaea gescheitert. Doch die Mehrzahl der anderen Provinzen hat sie zumeist unmittelbar gehört und sich danach gerichtet, so wie auch Judaea nach der Katastrophe des Bar Kochba-Aufstandes. Die Vorstellung von einer stets möglichen militärischen Intervention muss man sich immer vor Augen halten. Erst vor diesem Hintergrund sind all die anderen Kommunikationsformen zwischen Herrschenden und Beherrschten zu sehen, die wir in den römischen Provinzen ausmachen.

${ }^{17}$ Dazu unter Einschluss zahlreicher neuer Dokumente Eck, Rom und Judaea (Anm. 7), $113 \mathrm{ff}$.

${ }^{18}$ Höchstens Germania inferior wies im 1. Jahrhundert eine im Verhältnis zum Territorium vergleichbare Truppenstärke auf.

${ }^{19}$ W. Eck, Rom herausfordern: Bar Kochba im Kampf gegen das Imperium Romanum. Das Bild des Bar Kochba-Aufstandes im Spiegel der neuen epigraphischen Überlieferung (Rom 2007).

${ }^{20}$ PIR $^{2}$ I 576; P 1042; CIL XI 5212 = ILS 1058; W. Eck, 'The Bar Kokhba revolt: The Roman point of view', Journal of Roman Studies 89 (1999), 76 ff. 
Ohne diese selbstverständliche militärische Basis der Herrschaft, ohne die Möglichkeit der Aktivierung der Militärmacht hätten auch manche der anderen Zeichen wohl weniger oder gar keine Wirkung ausüben können. In gewisser Weise verwiesen alle anderen Zeichen immer wieder auf Roms militärische Überlegenheit zurück.

Diese zeigte sich in allen Provinzen und vor allem in der Umgebung des Statthalters, aber auch bei den kaiserlichen Prokuratoren. Ihr Personal war zu einem erheblichen Teil, bei den kaiserlichen Legaten sogar ausschließlich, dem Heer entnommen. ${ }^{21}$ Daneben gab es die Liktoren mit ihren Rutenbündeln und Beilen, die auch in der Kaiserzeit noch die senatorischen Amtsträger begleiteten. Den beiden Prokonsuln von Africa und Asia standen zwölf Liktoren zu, den anderen Prokonsuln sechs, während sich alle kaiserlichen Legaten mit fünf begnügen mussten. ${ }^{22}$ Das galt auch für den Statthalter von Judaea seit dem Jahre $66 \mathrm{n}$. Chr. Der vom syrischen Legaten abhängige praefectus aber konnte nur Soldaten zu seiner Begleitung aufbieten. Die Liktoren haben zwar die Entscheidungsgewalt des Vertreters Roms über Leben und Tod am klarsten ausgedrückt, doch dass sie während der Kaiserzeit wie früher die Todesurteile der Statthalter vollstreckten, ist eher unwahrscheinlich. Neben der großen Zahl von Soldaten in den meisten Provinzen wirkten die Liktoren eher wie eine symbolische Staffage, obwohl Josephus König Agrippa in seiner Rede an die aufständischen Juden im Jahr $66 \mathrm{n}$. Chr. nicht ohne Grund sagen lässt, die 500 Städte Asiens beugten sich, obwohl sie nicht besetzt seien, ehrfurchtsvoll vor einem Statthalter und den (pro)konsularen Rutenbündeln. ${ }^{23}$ Dies entspricht halbwegs dem äußeren Schein in der Provinz Asia. Doch in den meisten Provinzen waren die sichtbaren Zeichen römischer Herrschaft weitgehend durch das Militär geprägt. Gerade dies wird in Caesarea deutlicher sichtbar, als wir es bisher kannten, und zwar in der Ausgestaltung des Amtsitzes des Statthalters, wie es die Ausgrabungen in dieser

${ }^{21}$ Dazu R. Haensch, Capita provinciarum. Statthaltersitze und Provinzialverwaltung in der römischen Kaiserzeit (Mainz 1997), 713 ff.

${ }^{22}$ H.M. Cotton, 'Cassius Dio, Mommsen and the quinquefascales', Chiron 30 (2000), 217 ff.; Zeugnisse für Liktoren in den verschiedenen Provinzen beispielweise in ILS 1914; AE 1933, 265 = IPergamon III 67; ILS 1913 = IEph III 712; ILS $4056+$ AE 1939, 4 = P.M. Fraser, Samothrace II 1: The Inscriptions on Stone (New York (1960), Nr. 53.

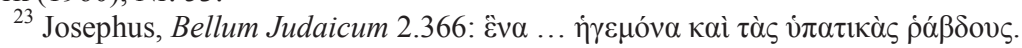


Stadt seit dem Ende der 80er Jahre des vergangenen Jahrhunderts klar gezeigt haben.

Schon aus der Apostelgeschichte konnte man entnehmen, dass der ritterliche praefectus den Palast des Königs Herodes in Caesarea übernommen hatte. Denn als der Apostel Paulus von Soldaten der in Jerusalem stationierten Kohorte als Gefangener nach Caesarea gebracht wurde, befahl der damalige Präfekt Felix, Bruder des großen

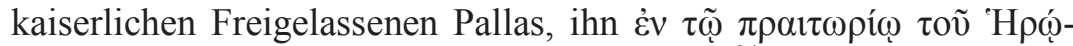
Sov zu internieren, im Praetorium des Herodes. ${ }^{24}$ Das ist natürlich die römische Terminologie für den Amtssitz des höchsten römischen Repräsentanten in der Provinz, aber der Verfasser der Apostelgeschichte war sich bewusst, auf wen der Bau zurückging. Bei den Ausgrabungen in Caesarea wurde auch das im Süden der Stadt liegende, ins Meer vorspringende Kap ausgegraben; die repräsentativen baulichen Strukturen, die man dabei fand, wurden trotz weitgehender Zerstörung durch das Meer sogleich als der Palast des Herodes erkannt. ${ }^{25}$ Diesen Komplex hat, wie man der Bemerkung der Apostelgeschichte entnehmen kann, der Vertreter Roms übernommen. Wenn somit die Interpretation des Komplexes als Palast des Herodes zutreffend ist, dann hat man damit auch den Amtssitz des Vertreters Roms identifiziert. Diese Identifikation wurde aber erst definitiv gesichert, als im Lauf der Ausgrabungen auch die nach Osten an das Kap anschließenden Bereiche erforscht wurden und dabei einige inschriftliche Dokumente zu Tage kamen, die im Folgenden zu besprechen sind.

Bei den Grabungen deckte man einen großen Gebäudekomplex auf, der unmittelbar an das von Herodes erbaute Stadium anschloss (Abb. 1). Er erstreckte sich über mindestens 250 Meter von West noch Ost, also vom Meer hinein ins Land, während die Nord-SüdAusdehnung sicher nicht weniger als 100 Meter betrug. Doch ist dies nicht mit letzter Sicherheit zu sagen, da im Süden größere Teile der Anlage vom Meer weggespült wurden. Der gesamte Komplex besteht aus dem auf Meereshöhe gelegenen Vorsprung mit einem Peris-

${ }^{24}$ Acta apostolorum 23.35 .

${ }^{25}$ B. Burrell, 'Palace to praetorium: the romanization of Caesarea', in: A. Raban - K.G. Holum (Hrsg.), Caesarea Maritima: a Retrospective After Two Millennia (Leiden 1996), 228 ff.; dies., 'Two inscribed columns from Caesarea Maritima', Zeitschrift für Papyrologie und Epigraphik 99 (1993), 287 ff. = AE 1993, 16191624. 
tyl und einem höher gelegenen zweiten Bereich. Innerhalb dieser weit ausgedehnten Strukturen erhob sich ein großer Innenhof, um den sich zahlreiche Räumlichkeiten erstreckten. Im Osten fanden sich drei Räume, die auf unsere Frage nach der Präsenz von Soldaten in der nächsten Umgebung des Statthalters und damit nach der Identifikation des Gebäudes als Praetorium eine klare Antwort geben.

Diese drei epigraphischen Zeugnisse wurden sämtlich in situ gefunden. Zum einen wurde in einem Raum an der südlichen Seite des Zugangs zum Innern des Palastes ein Mosaik mit folgendem Text aufgedeckt: ${ }^{26}$

Spes bona adiutoribus officii custodiarum

Eine glückliche Zukunft den Hilfsfunktionären im Büro der Gefängnisverwaltung

An dieser Stelle lag somit ein Gefängnistrakt, in dem Soldaten ihren Dienst taten. Denn obwohl der Text nicht direkt von Angehörigen des Militärs spricht, wissen wir aus anderen Zeugnissen, dass die hier beschriebene Aufgabe von Soldaten übernommen wurde. Ganz direkt ergibt sich die Anwesenheit von Militärs aus einem weiteren Mosaik, das in einem Raum nördlich des Hauptzugangs aufgedeckt wurde. Der Text dieser Mosaikinschrift lautet: ${ }^{27}$

Sanct [o] Genio fru[m] entarioru[m] omnia felicia

Der heiligen Kraft der frumentarii ein glückliches Gelingen

In diesem Raum müssen sich also über längere Zeit hinweg mehrere frumentarii versammelt haben, die generell aus den Legionen genommen und im Gerichtswesen herangezogen wurden. Bedeutsam ist, dass hier offensichtlich eine ganze Gruppe dieser Spezialsoldaten in der Nähe des Statthalters Dienst tat. Der dritte Text kam nicht weit von dem eben besprochenen zu Tage. Er steht auf einer runden, etwa $60 \mathrm{~cm}$ hohen Säule, die in einem Raum unmittelbar neben einem Tisch lag, der seinerseits an der Rückwand des Raumes steht, welcher sich direkt an die Außenwand des herodianischen Stadiums anlehnt. Dieser Text lautet: ${ }^{28}$

${ }^{26}$ H.M. Cotton - W. Eck, 'Governors and their personnel on Latin inscriptions from Caesarea Maritima', in: Proceedings of the Israel Academy of Sciences and Humanities VII No. 7 (Jerusalem 2001), $230 \mathrm{ff}$.

${ }^{27}$ Cotton - Eck, Governors (Anm. 26), $232 \mathrm{ff}$.

${ }^{28}$ Cotton - Eck, Governors (Anm. 26), 215 ff. 
Cl(audius) Severus cust(os) sc(olae) (centurionum) s(ua) p(ecunia) f(ecit)

Claudius Severus, Verwalter und Aufseher des Versammlungslokals der Zenturionen, hat (die Statue) aus eigenen Mitteln aufgestellt

Der Text sagt klar, dass der Raum, in dem die Säulenbasis stand und auf der sich vermutlich eine Geniusstatue erhob, einer Gruppe von Centurionen als Dienst- und gleichzeitig als Clubraum diente. Die Centurionen stammten aus den beiden Legionen der Provinz und waren zu besonderen Diensten nach Caesarea abgeordnet worden.

Damit sind drei Gruppen von Militärangehörigen innerhalb des statthalterlichen Praetoriums bezeugt. Alle hatten ihre Funktion im Verbund der administrativ-jurisdiktionellen Aufgaben des Statthalters. Sie trugen auch während ihres Dienstes Uniform, d.h. sie waren stets als Militärs zu erkennen, nicht anders als etwa die singulares, die als berittene Leibwache des Legaten dienten, oder die stratores, die für die Reitpferde des Legaten zuständig waren. Nach Ulpian durfte der Prokonsul diese stratores nicht etwa aus seiner eigenen familia nehmen, er hatte dazu vielmehr Soldaten heranzuziehen. ${ }^{29}$ Alle diese militärischen Chargen hielten sich in der unmittelbaren Umgebung des Statthalters auf, ihre Büros lagen an dem Zugangsweg zum Innern des Praetoriums. Jeder Besucher schritt an ihnen vorbei, ebenso an den Soldaten, die speziell für die Bewachung des Zugangs abgeordnet waren. Die aus dem Heer abkommandierten Soldaten waren somit nicht nur für den Vertreter Roms tätig, sie waren auch jederzeit sichtbar und prägten damit den Charakter der Herrschaft für alle diejenigen, die mit dem Statthalter zu tun hatten. Hiervon gab es sicherlich nicht wenige, denn der Statthalter war die Instanz, die Recht zu sprechen hatte; in den Schriften des Neuen Testaments erscheinen die Präfekten ausschließlich in dieser Funktion. Stets wird dabei auf das $\beta \tilde{\eta} \mu \alpha$ verwiesen, das Tribunal, ${ }^{30}$ auf dem der Amtsstuhl stand, lateinisch bezeichnet als sella curulis, auf dem der Richter Platz nahm, um seine Tätigkeit zu beginnen - eine geradezu typische Handlung für den Statthalter als Richter, wie auch Plinius

\footnotetext{
${ }^{29}$ Digesta 1.16.4.1.

${ }^{30}$ Matthäus 27.19; Johannes 19.13; Acta apostolorum 18.16 f.; 25.6.7. Vgl. beispielsweise auch P.Fouad I 21; ferner Haensch, Capita (Anm. 21), 82, 155, 170, 210 f., $232 \mathrm{ff} ., 259,324,355 \mathrm{f}$.
} 
der Jüngere in einem beiläufigen Satz bezeugt. ${ }^{31}$ Für weite Bereiche der Jurisdiktion, auch der Rechtssprechung in Zivilangelegenheiten, war der Provinzgouverneur allein zuständig. Symptomatisch ist dafür das Archiv der Jüdin Babatha, deren Dokumente in den Höhlen von Nahal Hever gefunden wurden, wohin sie sich während des Aufstandes des Bar Kochba mit anderen Frauen geflüchtet hatte. Für Babatha, also eine einzige Person, wurden in den wenigen Jahren zwischen 124 und 132 sieben Schriftstücke abgefasst, die sich auf Verfahren vor dem Statthalter in der Provinz Arabia bezogen, in der Babatha lebte. ${ }^{32}$ Das Archiv der Frau gibt keine Hinweise, die es erlauben würden, sie als einen untypischen Sonderfall zu betrachten. Sie verkörpert wohl den Durchschnitt der Provinzbewohner, die immer wieder der Hilfe des Statthalters bedurften. Damit aber lässt sich ermessen, wie viele Personen sich an den Vertreter Roms wandten und somit auch seine Umgebung erlebten und deren Gepräge in sich aufnahmen.

Bei allen Gerichtsverhandlungen, aber auch bei allen anderen öffentlichen Auftritten des Gouverneurs war Militär stets in der Umgebung des Statthalters präsent - in Caesarea und ebenso an vielen anderen Orten in der Provinz. Wenn Plinius der Jüngere während seiner Tätigkeit in der Provinz Pontus et Bithynia von der Ableistung der vota, dem Eid auf den Kaiser, oder von den Opfern zum dies imperii Traians spricht, dann geschieht dies stets in Gegenwart von Provinzialen und Soldaten, obgleich in dieser Provinz keine Legion stationiert war. ${ }^{33}$ Genau dieselbe Konstellation schildert Tertullian in Bezug auf den proconsul Africae in Carthago: Die votorum nuncupatio findet zuerst im Lager, dann auf dem Capitolium statt; beide Male wird der Prokonsul auch von Soldaten begleitet. ${ }^{34}$ Und Ähnliches lässt sich in Judaea schon unter dem Regime der Präfekten beobachten. Als Porcius Festus in Caesarea König Agrippa und dessen Schwester Berenike empfing, um ihnen Paulus vorzuführen, waren nicht nur die vornehmsten Leute der Hauptstadt in der Empfangshal-

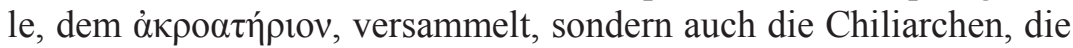

${ }^{31}$ Plinius minor, Epistulae 10.81.4: Ubi cum sedissem cogniturus... . Vgl. dazu die Darstellungen bei T. Schäfer, Imperii insignia: sella curulis und fasces. Zur Repräsentation römischer Magistrate (Mainz 1989).

${ }^{32}$ N. Lewis, The Documents from the Bar-Kokhba Period in the Cave of Letters: Greek Papyri (Jerusalem 1989).

${ }^{33}$ Plinius minor, Epistulae 10.52 f.; 100 f.; 102 f.

${ }^{34}$ Tertullian, De corona 12.3 . 
Befehlshaber der Militäreinheiten, die dem Präfekten unterstanden. ${ }^{35}$ War der Statthalter auf Reisen, dann geschah dies in der Begleitung seiner Leibwache und anderer Einheiten. Dabei führten sie nicht nur ihre Waffen mit sich, sondern auch ihre Feldzeichen, die zeigen sollten, wer der Befehlshaber aller Truppen war, denn an den signa waren die Porträts des Kaisers angebracht. Die Truppen sollen auf diese Weise die militärische Macht, aber auch den politischen Willen des Reiches repräsentieren. Das Mitführen der Feldzeichen und der Kaiserbilder war nicht in das Belieben des einzelnen Kommandeurs gestellt, sondern inhärenter Bestandteil des Auftrags der Truppen in allen Provinzen.

In diesen Kontext ist meines Erachtens eine recht bekannte Szene aus Judaea einzuordnen, die sich unter Pontius Pilatus abgespielt hat. ${ }^{36}$ Josephus berichtet von einem irritierenden Vorfall, den dieser Präfekt ausgelöst hat, denn er ließ Truppen, die nach Jerusalem ins Winterquartier gingen, ihre Feldzeichen mitsamt den dort angebrachten Kaiserbildern mitführen. ${ }^{37}$ Seine Vorgänger hatten dies, so jedenfalls der jüdische Historiker, nicht getan. Wenn man dem Autor glauben darf, stand hinter dem Vorgehen durchaus die Absicht des Präfekten, die jüdische Bevölkerung zu provozieren. Allerdings kann man bezweifeln, ob Josephus die Absicht des Pilatus richtig gedeutet hat, da nach seinem Bericht die Feldzeichen nachts und verhüllt in die Stadt gebracht wurden, so dass die Bilder also nicht unmittelbar gesehen werden konnten. Am nächsten Morgen hätte jedoch die Nachricht über die Anwesenheit der Feldzeichen in Jerusalem unter den Juden höchste Unruhe ausgelöst, die erst nach mehreren Tagen höchster Anspannung gelöst werden konnte, als Pilatus schließlich den Befehl gab, die Feldzeichen wieder aus Jerusalem zu entfernen. Die Unruhe, ja Revolte der Juden in Jerusalem wurde offensichtlich von den Kaiserbildern ausgelöst, die an den Feldzeichen angebracht waren. Da das göttliche Gesetz, so wie es von manchen Juden verstanden wurde, die Herstellung jeglicher Abbildungen von Menschen verbot, hätte folglich die Anwesenheit dieser Bilder den heiligen Status der Stadt verletzt. Dies hätte noch weit gravierendere Folgen gehabt, wenn Pilatus die Feldzeichen tatsächlich im Tempel hätte auf-

\footnotetext{
${ }^{35}$ Acta apostolorum 25.23.

${ }^{36}$ Siehe zum Folgenden Eck, Rom und Judaea (Anm. 7), 55 ff.

${ }^{37}$ Josephus, Bellum Judaicum 2, 169 ff.; Antiquitates Judaicae 18.55 ff. Siehe dazu auch Eusebius, Historia ecclesiastica 2.6.4.
} 
stellen lassen, wie es nach Philo, den der Kirchenhistoriker Eusebius zu Beginn des 4. Jahrhunderts zitiert, der Fall gewesen sein soll. ${ }^{38}$ Von Josephus und Philo wurde der Vorfall also offensichtlich als bewusste Provokation durch Pilatus verstanden, da der Präfekt nicht gewillt gewesen sei, auf die religiösen Gefühle der Juden Rücksicht zu nehmen.

Man fragt sich nur, weshalb Pilatus den Truppen befahl, die Feldzeichen verhüllt und zudem während der Nacht nach Jerusalem zu bringen, wenn er damit bewusst die Absicht verfolgte, die Bevölkerung in ihren religiösen Gefühlen zu provozieren. Eine bessere Gelegenheit zur Provokation als den Einmarsch von Truppen bei Tag mit den weithin sichtbaren Zeichen hätte es doch kaum geben können! Man wird hier zwar kaum zu einer eindeutigen Antwort kommen, da wir keine andere Überlieferung als die bei Josephus und Philo haben. Doch beide Autoren - und das sollte man bei der Beurteilung dieses und ähnlicher Ereignisse nicht vergessen - sind ganz offen parteiisch und keine objektiven Beobachter, die Rom und seine Sicht höchstens partiell verstanden haben oder auch nicht verstehen wollten. Nimmt man jedoch den sehr konkreten Hinweis auf den Transport der verhüllten Feldzeichen in der Nacht ernst, dann drängt es sich geradezu auf, eine ganz andere Motivation zu vermuten, dass nämlich Pilatus die jüdischen Vorstellungen soweit nur irgend möglich schonen wollte, indem die Feldzeichen während des Transports für niemanden sichtbar waren. Nur darf man nicht vergessen, dass Pontius Pilatus Römer war, vermutlich ein machtbewusster Römer, der das, was nach seiner Sicht zur römischen Herrschaft gehörte, nicht preisgeben wollte. Dass römische Truppen über längere Zeit hinweg ohne diese Feldzeichen mit ihrem Bedeutungsinhalt ihren Dienst in Jerusalem versehen sollten, entsprach wohl nicht dem, was er als selbstverständliche Ausdrucksform römischer Macht empfand. Zumindest in ihrer Kaserne in Jerusalem sollten diese Zeichen und Bilder präsent sein. Mag sein, dass sich darin ein Verständnis römischen Stolzes und römischen Selbstbewusstseins manifestierte, mit dem Pilatus sich von seinen Vorgängern abhob. Dass er zunächst trotz der massiven Proteste der Juden nicht zurückweichen wollte, es am Ende aber dennoch tat, könnte diese Interpretation stützen. Aus seiner Haltung spricht deutlich ein zumindest partielles Unverständ-

\footnotetext{
${ }^{38}$ Eusebius, Demonstratio evangelica 8.2.123.
} 
nis gegenüber den Vorstellungen der Mehrheit der Bevölkerung, ebenso jedoch wohl auch seine Sicht von der Art und Weise, in der sich Rom in seinem Heer repräsentierte, was wiederum von Seiten eines Teils der Juden konzessionslos als unerträglich angesehen wurde.

Doch zurück nach Caesarea: Im Amtssitz des Statthalters konzentrierte sich vor Ort die römische Herrschaft. Es ist deshalb auch nicht verwunderlich, wenn gerade die Statthalter im Inschriftenmaterial der Stadt besonders häufig vertreten sind und wenn vor allem sie als die Vertreter Roms mit Statuen geehrt wurden. Nicht wenige dieser Statuen waren auf fast zwei Meter hohen Säulen postiert, von denen manche innerhalb des Praetoriums aufgestellt waren. Sie repräsentierten dort die Kontinuität der Macht durch die Repräsentanten des Kaisers, wobei auch anderswo die Gouverneure statuarisch vorgeführt wurden. So hat sich während der Ausgrabungen eine Konsole gefunden, die in ein Bauwerk eingelassen war und eine Statue getragen hatte. Die Ehrung galt einem Gaius Iulius Commodus Orfitianus, Suffektkonsul im Jahr 157 und Statthalter in Syria Palaestina ab $161 .^{39}$ Dieser Text zeigt eine der für manche Provinzbewohner erfreulichen Seiten römischer Herrschaft, wie sie durch die kaiserlichen Legaten ausgeübt werden konnte: Commodus hatte Valerius Martialis, einem Bewohner von Caesarea und Sohn eines ehemaligen primipilus, einen Militärtribunat in einer Legion, vermutlich in der Provinz selbst, verschafft und ihm damit die Chance eröffnet, einen weiteren Schritt zum Eintritt in die Reichsaristokratie zu tun. Die Antwort des jungen Ritters in diesem Prozess der Kommunikation war die Ehrung dessen, der seinen Aufstieg ermöglicht hatte. In der Inschrift, die er unter der Statue seines Gönners anbringen ließ, wird sehr deutlich gemacht, worin sich die Herrschaftsfunktion des Legaten ausgewirkt hatte. Zwischen der Formel ob m(erita) steht ex secunda militia, also ein Hinweis darauf, dass Valerius Martialis seine zweite ritterliche Dienststellung erfolgreich abgeschlossen hatte. ${ }^{40}$ Deutlicher hätte man nicht zeigen können, durch welches Handeln sich die Teilhabe des Statthalters an der Herrschaft konkret ausgewirkt hatte.

Caesarea lässt uns aber als bisher einzige Stadt im gesamten Imperium nicht nur den Sitz des Statthalters kennenlernen, in dem sich

\footnotetext{
${ }^{39}$ Siehe RMD IV 275 (28. September 157) und ibid. III p. 246 zu Anm. 55.

${ }^{40}$ Cotton - Eck, Govenors (Anm. 26).
} 
die römische Herrschaft in vielfältiger Form manifestiert hat; vielmehr konnte hier auch der Sitz des Finanzprokurators identifiziert werden. In allen anderen Fällen, in denen der Statthalter am selben Ort wie der Prokurator residierte, ist höchstens eines der beiden vorauszusetzenden praetoria gefunden worden, oft auch gar keines. ${ }^{41}$ Caesarea aber zeigt beide und lässt deutliche Differenzen erkennen: Während die Statthalterresidenz sogleich ab 6 n. Chr. den alten Königspalast des Herodes übernahm, wurde das Praetorium des Finanzprokurators erst unter Vespasian geschaffen. Es ist sicher kein Zufall, dass man dieses neue Praetorium nicht mit dem Sitz des Legaten verband, obwohl beide kaiserliche Funktionsträger waren und im Namen des Kaisers die römische Macht vertraten, sondern dass für den Finanzbeauftragten des Kaisers ein eigenes Praetorium geschaffen wurde. An der weitgeschwungenen Bucht, die sich zwischen dem südlichen Kap und dem durch Herodes neu geschaffenen Hafen erstreckte, wurde das Praetorium des Finanzchefs der Provinz in einer Distanz von circa 400 Metern vom Statthalterpalast angelegt, nahe am Hafen. ${ }^{42}$ Dies war kein Zufall. Schließlich wurden über den Hafen viele Güter angeliefert, die durch den Prokurator an die provinziale Verwaltung und insbesondere an das Heer ausgeliefert wurden, unter anderem Getreide, Waffen und Metalle. So erstaunt es nicht, dass man im Hafen der Stadt insgesamt sechs Bleibarren gefunden hat, die aus den met(alla) Dard(anica) in Obermösien stammten und unter Domitian nach Judaea geliefert worden waren. ${ }^{43}$ Die Lage des Praetoriums ist damit ganz deutlich funktional bedingt, doch die Trennung seines Amtssitzes von dem des Statthalters sollte auch die Unabhängigkeit dieses ritterlichen Amtsträgers vermitteln und eine spezifische Tätigkeit gegenüber der Öffentlichkeit der Provinz herausstellen.

Das prokuratorische Praetorium (Abb. 2) ruht auf vier gewaltigen gewölbten Räumen, die zumindest ursprünglich als Speicher gedient hatten; in einem wurde später die Versammlungsstätte einer Mithrasgemeinde eingerichtet. $\mathrm{Zu}$ Beginn war das gesamte Praetorium eben-

\footnotetext{
${ }^{41}$ Siehe dazu Haensch, Capita (Anm. 21), passim.

${ }^{42}$ Siehe dazu vorläufig J. Patrich, 'The warehouse complex and governor's palace (areas KK, CC, and NN, May 1993 - December 1995)', in: K. G. Holum - A. Raban - J. Patrich (Hrsg.), Caesarea Papers 2 (Portsmouth 1999), 70 ff.

${ }^{43}$ A. Raban, 'The lead ingots from the wreck site (area K8)', in: Holum - Raban - Patrich, Caesarea Papers (Anm. 42), 179 ff. = AE 1999, 1683.
} 
falls zum Meer hin ausgerichtet gewesen, doch bald erfolgte eine Umorientierung der Fassade nach Osten, so dass von nun an der $\mathrm{Zu}-$ gang vom Cardo maximus aus erfolgte, nicht anders als auch beim statthalterlichen Praetorium. Der Zugang wurde monumental mit eleganten Säulen ausgestaltet, und im Zentrum wurde eine Halle mit einer Apsis erbaut, die vermutlich für die öffentlichen Auftritte des Prokurators genutzt wurde, was in seinem Fall wohl vornehmlich Gerichtsverhandlungen meinte. Während sich im Norden des Gebäudes ein Archivraum befand, stand am südlichen Rand des Komplexes eine große Latrine bereit, was auf einen nicht geringen Publikumsverkehr hindeutet. Der gesamte Bau war ebenso auf Repräsentation ausgelegt wie das Statthalter-Praetorium: Das Publikum sollte offensichtlich beeindruckt werden.

Ein deutlicher Unterschied scheint allerdings in der Größe der beiden administrativen Komplexe zu liegen. Denn während der Amtssitz des Statthalters sich schätzungsweise über rund $25.000 \mathrm{~m}^{2}$ oder auch mehr erstreckte, musste der Prokurator sich offensichtlich mit weniger als der Hälfte, vielleicht sogar nur mit rund $8.000 \mathrm{~m}^{2}$ begnügen - wenn der jetzt zugängliche Befund die volle Ausdehnung erkennen lässt. ${ }^{44}$ Denn beim archäologischen Befund ist einzubeziehen, dass irgendwann im 4. Jahrhundert der ursprüngliche Statthaltersitz aufgegeben und in den ehemaligen Prokuratorenpalast verlegt wurde. Dabei wurde sicherlich manches tiefgreifend verändert, wie es unter anderem die Mosaikböden in verschiedenen Räumen nahelegen, die fast alle aus der Zeit ab dem 4. Jahrhundert stammen, ausgenommen zwei lateinische, die in die Epoche der Finanzprokuratoren gehören. Es ergibt jedenfalls einen Sinn, wenn der Dienstsitz des Prokurators tatsächlich von seiner Ausdehnung her und damit in einem nicht unwesentlichen Aspekt von öffentlicher Repräsentation deutlich dem Praetorium des kaiserlichen Legaten nachgeordnet gewesen wäre. Vor allem weit ausgedehnte Empfangsräume und die großen Peristylia finden sich vornehmlich im Praetorium des Statthalters. Die Räumlichkeiten beim Prokurator sind wesentlich kleiner, weniger aufwendig und offensichtlich nicht auf so zahlreiche Personen, die gleichzeitig anwesend waren, ausgelegt.

Die sonstigen Formen der Prestigedemonstration aber wurden auch im Prokuratoren-Praetorium angewandt, um zu zeigen, dass

\footnotetext{
${ }^{44}$ Für eine endgültige Beurteilung wird man den Final Report abwarten müssen.
} 
hier der zweite hohe Vertreter des Kaisers seine Aufgaben versah. Wie man aus den vielen Inschriften erschließen kann, waren auch hier die Hallen und Räume mit Statuen ausgestattet, wobei - soweit eine Rekonstruktion möglich ist - vor allem die Amtsinhaber in stattlicher Zahl vertreten waren. Nicht weniger als etwa 40 entsprechende Inschriften oder Inschriftenfragmente sind in dem Bereich des Praetorium gefunden worden, die zu Porträtbüsten oder auch lebensgroßen Statuen gehörten, die auf kleinen Sockeln oder auf Säulen ähnlich wie im Statthalter-Praetorium aufgestellt waren. Eine dieser runden Statuenbasen bezeugt eine Ehrung des Furius Timesitheus, des späteren Prätorianerpräfekten und Schwiegervaters Kaiser Gordian III, ${ }^{45}$ eine weitere verweist auf einen Prokurator von Syria Palaestina des frühen 3. Jahrhunderts, Valerius Valerianus. ${ }^{46}$ Noch zahlreicher waren wohl Porträts, unter denen kleinere Inschriften angebracht waren, die nur den Namen und die Titulatur des Geehrten nannten, während andere Inschriften mit einem vollen cursus honorum auf größere gemauerte Sockel verweisen, von denen Statuen auf die Besucher herabblickten. ${ }^{47}$ Doch stets ist es die offizielle Amtsstellung und die damit verbundene Macht, die hier ihren Ausdruck fand.

Aber nicht nur die Leiter der Fiskalverwaltung wurden hier geehrt; gelegentlich öffneten sich diese Räume auch für andere, die nicht zu der absoluten Spitze der römischen Amtsträger gehörten, wie etwa für einen T. Flavius Callistus, einen Freigelassenen der Flavier, von dessen Ehrenstatue sich immerhin noch die Basis erhalten hat. ${ }^{48}$ Er wurde im Bereich des Praetorium von einem amicus, einem römischen Bürger, geehrt. Dies ist ein schwacher Abglanz dessen, was kaiserliche Freigelassene, die ebenfalls die römische Macht repräsentierten, im Raum einer Kolonie wie Caesarea darstellen konnten. Das oft machtvolle Handeln solcher liberti Augusti durch administrative Akte war in der Öffentlichkeit unmittelbar zu verfolgen. So besagt ein in Caesarea geschriebener Papyrus, der zufälligerweise bis nach Ägypten gelangte und so erhalten blieb, dass der

${ }^{45}$ C. M. Lehmann - K. G. Holum, The Greek and Latin Inscriptions of Caesarea Maritima (Boston 2000), Nr. 7: C. Furio Timesitheo proc(uratori) Aug(usti) Aur(elius) Iustinus (centurio) strat(or) eius.

${ }^{46}$ Lehmann - Holum, Inscriptions (Anm. 45), Nr. 4.

${ }^{47}$ Siehe dazu die zukünftige Publikation der Texte im Final Report. Vorläufig zu einigen der einschlägigen Texte vgl. Eck, Rom und Judaea (Anm. 7), $100 \mathrm{f}$.

${ }^{48}$ Lehmann - Holum, Inscriptions (Anm. 45), Nr. 2: T(ito) Flavio Aug(usti) liber(to) Callisto C. Aurunculeius amico suo h(onoris) c(ausa). 
kaiserliche Freigelassene Aelius Amphigethes im Jahr 152 in Caesarea im Tempel, $\dot{\varepsilon} v \tau \tilde{\omega} v \alpha \tilde{\omega}$, ein Verwaltungsverfahren zu Ende führte, und zwar im Auftrag des Prokurators Calpurnius Quintianus. ${ }^{49}$ Jeder konnte dem Verfahren folgen, da es öffentlich stattfand, doch in welchem Tempel genau es ablief, sagt der Papyrus nicht. Angesichts der schlichten Aussage $\dot{\varepsilon} v \tau \tilde{\omega}$ v $\alpha \tilde{\omega}$ könnte man jedoch vermuten, dass damit der mächtige Tempel für Augustus gemeint ist, den Herodes über dem inneren Hafen errichtet hatte. Jedenfalls ergibt sich aus diesem Zeugnis, dass die kaiserlichen Funktionsträger nicht nur innerhalb ihres administrativen Baukomplexes südlich des Hafens auftraten und Roms Macht und Zuständigkeit zeigten, sondern auch in der vollen Öffentlichkeit der Kolonie.

Was den Besuchern in beiden praetoria aufgefallen sein dürfte, war die Einheitlichkeit der Sprache, in der während der ersten drei Jahrhunderte der Kaiserzeit die Inschriften abgefasst waren: Latein dominierte überall. Die griechische Sprache scheint, jedenfalls für dieses dauerhafte Medium der Kommunikation, nicht benutzt worden zu sein, ganz im Gegensatz zu den Heimatstädten der Besucher in den anderen Teilen der Provinz. Nur beispielhaft sei auf zwei Inschriften auf Statuenbasen für Frau und Tochter des Tineius Rufus aus Scythopolis verwiesen, welcher Legat in Judaea war, als der Bar Kochba-Aufstand ausbrach. Die beiden Basen sind bisher die einzigen Zeugnisse für Ehrenstatuen von Mitgliedern der Reichsführungsschicht aus Scythopolis und sind in griechischer Sprache abgefasst, da sie von der Polis Scythopolis errichtet wurden. ${ }^{50}$ In Caesarea musste der Besucher aus den meisten anderen Städten der Provinz allein wegen der lateinischen Inschriften im öffentlichen Raum realisieren, dass er eine andere Welt betrat: Die Welt Roms, in der er zwar auch lebte, aber der er nur zum Teil zugehörte. Im Eingang zum Praetorium des Prokurators begrüßte den Besucher die lateinische Akklamation Feliciter. ${ }^{51}$ Nicht jeder wird geglaubt haben, der Glückwunsch werde sich auch für ihn erfüllen, da der Prokurator

${ }^{49}$ H. Maehler, 'Ein römischer Soldat und seine Matrikel', in: E. Kießling - H.A. Rupprecht (Hrsg.), Akten des XIII. Internationalen Papyrologenkongresses (München 1974), 241 ff.; J. Rea, 'Two legates and a procurator of Syria Palaestina', Zeitschrift für Papyrologie und Epigraphik 26 (1977), 217 ff., bes. 218 ff.; W. Eck, 'Ein Prokuratorenpaar von Syria Palaestina in P.Berol 21652', Zeitschrift für Papyrologie und Epigraphik 123 (1998), 249 ff.

${ }_{50}^{5}$ Die Publikation dieser Texte ist in Vorbereitung.

${ }^{51}$ Siehe oben Anm. 47. 
schließlich möglichst den Steuerertrag maximieren, nicht aber in erster Linie die humane Seite Roms repräsentieren sollte.

Freilich, die Masse der Bewohner der Provinz verstand die Sprache Roms, das Lateinische, wohl kaum. Nur rund 500 lateinische Inschriften sind bisher im heutigen Israel, welches in etwa der römischen Provinz Judaea/Syria Palaestina entspricht, gefunden worden, die fast ohne Ausnahme aus den ersten drei Jahrhunderten der Kaiserzeit stammen. ${ }^{52}$ Dagegen stehen mehrere Tausend griechischer Texte sowie zahlreiche hebräische, aramäische, syrische und nabatäische Zeugnisse. Außer in der colonia Caesarea und der colonia Aelia Capitolina wurde Latein fast nur von Angehörigen des Militärs oder Personen, die näher mit der herrschenden Macht verbunden sind, verwendet. Außerhalb der Städte waren es im Wesentlichen die Meilensteine, auf denen den Provinzialen Latein begegnete. Die wesentliche Aussage der Steine war den meisten wohl trotz der Unkenntnis der Sprache klar. Hier hatte der Herr der Welt Straßen erbauen lassen, die den Provinzialen dienen sollten, aber vornehmlich auch die Beherrschung des Landes ausdrückten. Für diese Botschaft brauchte man die Sprache des Herrschers selbst nicht zu verstehen, denn was der Reisende konkret wissen wollte, die Entfernung zum nächsten Zentrum oder die Anzahl der bereits zurückgelegten Meilen, erschien ohnehin in griechischer Sprache. So heißt es etwa auf einem Meilenstein an der Straße von Jerusalem nach Emmaus, das

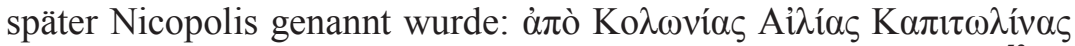

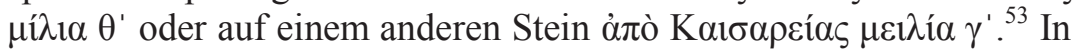
dieser marginalen Kleinigkeit zeigt sich die Pragmatik der Römer, die zwar immer dann, wenn es um Herrschaft und politisches Prestige ging, auf ihrer eigenen Sprache als Zeichen beharrten, sich aber ansonsten den Notwendigkeiten anpassten. Zwar hat sich nach allem, was wir wissen, kein Statthalter des Aramäischen als Sprache der Mehrheit der Bevölkerung bedient - das hat erst Mel GIBSON in seinem Film Passion of Christ geschafft, in dem er Pilatus mit den Mitgliedern des Hohen Rats in Jerusalem Aramäisch parlieren ließ -, doch die griechische Sprache, die auch die professionellen Schreiber beherrschten, wurde offensichtlich weithin verwendet und akzeptiert.

\footnotetext{
${ }^{52}$ Im Detail dazu Eck, Rom und Judaea (Anm. 7), 157 ff., bes. 186 ff.

${ }^{53}$ P. Thomsen, Zeitschrift des Deutschen Palästina-Vereins 40 (1917), 74f., Nr. 261; 77, Nr. 272; vgl. M. Fischer - B. Isaac - I. Roll, Roman Roads in Judaea II. The Jaffa-Jerusalem Roads (Oxford 1996), 294.
} 
Wiederum dienen die Dokumente Babathas sowie die einer anderen Jüdin, Salome Komaise, hierfür als Zeugnis. ${ }^{54}$ Wer immer mit der römischen Macht in Kontakt kommen wollte und und dies in schriftlicher Form tun musste, tat es auf Griechisch. ${ }^{55}$ Zur Darstellung der Herrschaft Roms aber war bis zum Ende des dritten Jahrhunderts Latein, die Sprache Roms, für jeden Funktionsträger zwingendes Erfordernis, was selbst die kaiserlichen Freigelassenen mit einschloss; konsequenterweise wurde die Inschrift unter der statuarischen Ehrung für Titus Flavius Callistus Augusti libertus in Caesarea in dieser Sprache abgefasst. ${ }^{56}$ Doch als gegen Ende des 5. oder zu Anfang des 6. Jahrhunderts ein comes Flavios Euelpidios, also ein hoher Amtsträger der spätantiken Verwaltung, ebenfalls in Caesarea ein Bauwerk erneuern ließ, wurde die Bauinschrift in griechischer Sprache abgefasst. ${ }^{57}$ Zwar nannte sich das Reich immer noch römisch, aber das Zentrum lag nun in Konstantinopel. Die Sprache dokumentiert diesen Wandel der Herrschaft.

Köln, Dezember 2007

${ }^{54}$ Lewis, Documents (Anm. 32) und A. Yardeni - B. Levine - C. Greenfield, Documents from the Bar Kokhba Period in the Cave of Letters: Hebrew, Aramic and Nabatean (Jerusalem 2002) sowie H. M. Cotton - A. Yardeni, Aramaic, Hebrew and Greek Documentary Texts from Nahal Hever and Other Sites (Oxford 1997).

${ }^{55}$ H.M. Cotton, 'The languages of the legal and administrative documents from the Judaean desert', Zeitschrift für Papyrologie und Epigraphik 125 (1999), 219231; dies., 'Survival, adaptation and extinction: Nabataean and Jewish Aramaic versus Greek in the legal documents from the Cave of Letters in Nahal Hever', in: L. Schumacher - O. Stoll (Hrsg.), Sprache und Kultur in der kaiserzeitlichen Provinz Arabia (St. Katharinen 2003), 133 ff.

${ }^{56}$ Siehe oben Anm. 47.

${ }^{57}$ Lehmann - Holum, Inscriptions (Anm. 45), Nr. 58. Zu anderen Formen der Kommunikation siehe den Sammelband von U. Peter - S. Seidlmayer (Hrsg.), Mediengesellschaft Antike? Information und Kommunikation vom Alten Ägypten bis Byzanz (Berlin 2006), darin: W. Eck, 'Herrschaft und Kommunikation in antiken Gesellschaften: Das Beispiel Rom', 11 ff. 


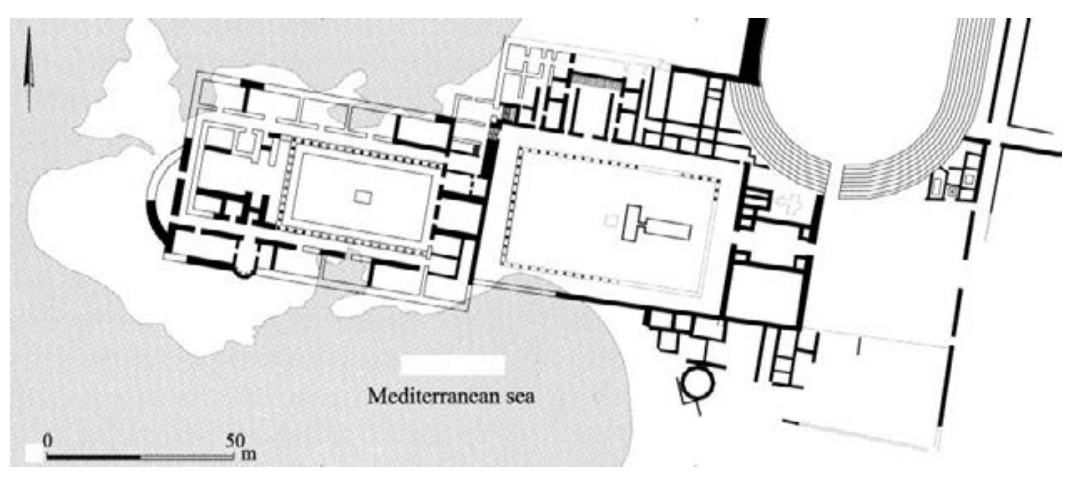

Abb. 1: Caesarea, Praetorium des Statthalters, nach Y. Porath, Hadashot Arkheologiyot:Excavations and Surveys in Israel 112 (2001) 40, Abb. 51.

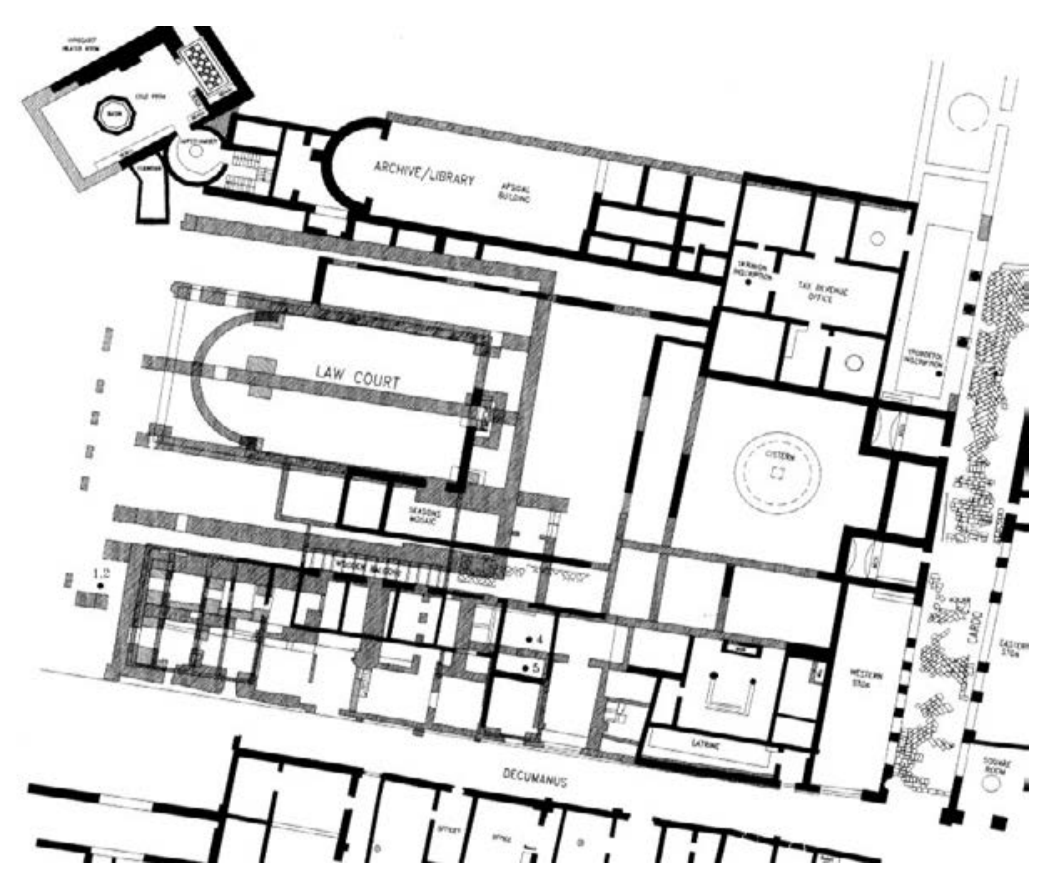

Abb.2: Caesarea, Praetorium des Prokurators, nach L. Di Segni - J. Patrich - K. G. Holum, 'A schedule of fees for official services from Caesarea Maritima, Israel', Zeitschrift für Papyriologie und Epigraphik 145 (2003), 299. 